\title{
Huge effect of arginine on survival in gastric cancer?
}

\author{
Kristoffer Lassen · Kim Mortensen • Ewen Griffiths • \\ Samir Johna $\cdot$ Magnus Nilsson
}

Received: 16 January 2014 / Accepted: 3 February 2014 / Published online: 13 February 2014

(C) Springer-Verlag Berlin Heidelberg 2014

To the Editors,

We have read with interest the paper published in your journal by Zhao et al. (2013). The authors present a single-centre, double-blinded RCT with a total of 73 patients undergoing gastrectomy for cancer. One group $(n=37)$ was given arginine-enriched enteral tube feeding for 7 days postoperatively. The paper presents an improved overall survival (OS) in the intervention group of median 41 months compared to 30.5 months in the control group. Furthermore, the proportion still alive after 5 years in the intervention group appears from the survival plot to be over $40 \%$, compared to less than $20 \%$ in the control group.

The results presented by Zhao et al. are staggering and would, if confirmed, more than offset the assumed benefits of perioperative chemotherapy or chemoradiation (Blum et al. 2013). Whilst numbers are small, the effect is overwhelming.

However, we have several major concerns with this paper.

K. Lassen $(\bowtie) \cdot$ K. Mortensen

Department of GI/HPB Surgery, University Hospital Northern

Norway, Tromsö, Norway

e-mail: lassen@unn.no

E. Griffiths

Department of Upper GI Surgery, University Hospitals

Birmingham NHS Foundation Trust, Queen Elizabeth Hospital,

Edgbaston, Birmingham B15 2WB, UK

\section{S. Johna}

Loma Linda University School of Medicine, 9961 Sierra Avenue, Fontana, CA 92335, USA

M. Nilsson

Department of Surgical Gastroenterology, Karolinska

University Hospital, Stockholm, Sweden
1. There is no description of how the randomisation sequence was generated and how it was protected. Neither is there any description of how, when and where allocation was performed or by whom. Importantly, there is no description of how blinding was achieved and whether it was complete. We are not offered any statistical calculations of sample size or power estimates.

2. The trial is, as far as we can assess, not registered on www.ClinicalTrials.gov and the register that has been used should be disclosed along with appropriate registration-ID.

3. To be able to assess whether the results could be caused by bias or randomisation breakdown, the authors must provide details of potentially cofounding variables in the two groups. The most obvious of which is the final histopathology from the resected specimens which is not mentioned at all. As chemotherapy appears not to have been used at all, simple variables such as depth of tumour invasion, tumour diameter, overall stage, lymph node status and surgical margin status must be provided to be sure the groups were not significantly different to begin with.

In light of our concerns regarding this paper, we are forced to view these results with grave scepticism.

Conflict of interest The authors declare no conflicts of interest.

\section{References}

Blum MA, Takashi T, Suzuki A, Ajani JA (2013) Management of localized gastric cancer. J Surg Oncol 107:265-270

Zhao H, Zhao H, Wang Y, Jing H, Ding Q, Xue J (2013) Randomized clinical trial of arginine-supplemented enteral nutrition versus standard enteral nutrition in patients undergoing gastric cancer surgery. J Cancer Res Clin Oncol 139:1465-1470 